\title{
Prenatally Diagnosed Left Atrial Appendage Aneurysm with Various Postnatal Imaging Investigations: A Case Report
}

\author{
Takeshi Sasaki $^{1}$, Yuki Kawasaki ${ }^{1}$, Yosuke Murakami ${ }^{1}$, Mitsuhiro Fujino ${ }^{1}$, Kae Nakamura ${ }^{1}$, \\ Yoko Yoshida $^{1}$, Tsugutoshi Suzuki ${ }^{1}$, Kyoichi Nishigaki ${ }^{1}$, and Eiji Ehara ${ }^{1}$ \\ ${ }^{1}$ Osaka City General Hospital
}

May 13, 2021

\begin{abstract}
A congenital left atrial appendage aneurysm (LAAA) is a rare cardiac malformation that is usually diagnosed in adulthood. It is rarely diagnosed prenatally. In most cases, surgical resection is recommended soon after the diagnosis has been made due to the risk of arrhythmia and thrombotic events. The present report describes a case of LAAA which was prenatally diagnosed and was asymptomatic postnatally. Imaging revealed the relation of the cardiac and airway structures around the LAAA in detail. The patient underwent surgical resection of the LAAA successfully at 7 months of age and is currently healthy at 5 years of age.
\end{abstract}

Prenatally Diagnosed Left Atrial Appendage Aneurysm with Various Postnatal Imaging Investigations: A Case Report

Takeshi Sasaki, MD, ${ }^{\text {a }}$ Yuki Kawasaki, MD, ${ }^{\mathrm{a}}$ Yosuke Murakami, MD, ${ }^{\mathrm{a}}$ Mitsuhiro Fujino, MD, ${ }^{\mathrm{a}}$ Kae Nakamura, MD, ${ }^{\text {a }}$ Yoko Yoshida, MD, ${ }^{\mathrm{b}}$ Tsugutoshi Suzuki, MD, PhD, ${ }^{\mathrm{b}}$ Kyoichi Nishigaki, MD, PhD, ${ }^{\mathrm{c}}$ Eiji Ehara, MD ${ }^{\mathrm{a}}$

${ }^{a}$ Department of Pediatric Cardiology, Osaka City General Hospital, Osaka, Japan.

b Department of Pediatric Arrhythmia, Osaka City General Hospital, Osaka, Japan.

${ }^{c}$ Department of Pediatric Cardiovascular Surgery, Osaka City General Hospital, Osaka, Japan.

Department of Pediatric Cardiology, Osaka City General Hospital Pediatric Medical Center, 2-13-22 Miyakojima-hondori, Miyakojima-ku, Osaka 534-0021 Japan

Corresponding Author: Takeshi Sasaki, MD

ORCID ID https://orcid.org/0000-0002-9174-6718

Department of Pediatric Cardiology, Osaka City General Hospital Pediatric Medical Center, 2-13-22 Miyakojima-hondori, Miyakojima-ku, Osaka 534-0021 Japan

E-mail:tsasaki.eve1224@gmail.com

Tel: $+81(6) 6929-1221$

Fax: $+81(6) 6929-0886$

Running head: Left atrial appendage aneurysm

Abstract

A congenital left atrial appendage aneurysm (LAAA) is a rare cardiac malformation that is usually diagnosed in adulthood. It is rarely diagnosed prenatally. In most cases, surgical resection is recommended soon after 
the diagnosis has been made due to the risk of arrhythmia and thrombotic events. The present report describes a case of LAAA which was prenatally diagnosed and was asymptomatic postnatally. Imaging revealed the relation of the cardiac and airway structures around the LAAA in detail. The patient underwent surgical resection of the LAAA successfully at 7 months of age and is currently healthy at 5 years of age.

Keywords

left atrial appendage aneurysm, fetal echocardiography, prenatal diagnosis, computed tomography, angiography

\section{INTRODUCTION}

A congenital left atrial appendage aneurysm (LAAA) is a rare congenital condition that is usually diagnosed in the second to fourth decade of life (1-3). Cases of prenatal LAAA have been rarely reported $(4,5)$. The clinical manifestations include palpitations, dyspnea, arrhythmia, and thromboembolic complications; however, some patients may be asymptomatic in the first decade of life $(2,3)$. The present report describes the prenatal echocardiographic findings in a patient with an LAAA, her postnatal clinical course, and the findings on various imaging investigations performed postnatally.

Case report

An abnormal echo-free space was detected on a regular fetal cardiac scan at 19 weeks of gestation. The fetus was then referred to our hospital owing to a suspicion of cor triatriatum. At 31 weeks of gestation, the three-vessel view on fetal echocardiography revealed an abnormal chamber adjacent to the main pulmonary artery; "to and fro" communication between that chamber and the normal-sized left atrium (LA) was noted (Figure 1). The four-chamber view showed no abnormalities, and pulmonary venous return was normal. The left atrial appendage (LAA) was not identified. Thus, the fetus was diagnosed with a left atrial appendage aneurysm (LAAA). The size of the LAAA was found to be $14 \mathrm{~mm} \times 16 \mathrm{~mm}$ on fetal echocardiography.

The female baby born at 38 weeks of gestation with a weight of $2.5 \mathrm{~kg}$ was asymptomatic. Transthoracic echocardiography confirmed the diagnosis of an LAAA that measured $30 \mathrm{~mm} \times 18 \mathrm{~mm}$. The communication between the LAAA and LA was not restrictive, and no thrombus formation was identified in the LAAA. The LAAA was visualized just adjacent to the left superior pulmonary vein and left coronary artery, without any sign of compression of those structures (Figure 2). Electrocardiography revealed a normal sinus rhythm without ST-T depression. Three-dimensional computed tomographic images depicted a huge, well-defined LAAA without compression of the left bronchus, left upper pulmonary vein, and left coronary artery (Figure 3). Cardiovascular magnetic resonance (CMR) imaging performed at 15 days of age confirmed the presence of a giant aneurysm of the LAA communicating with a normal-sized LA. Surgical resection was scheduled in late infancy since the baby was asymptomatic and the clinical examination was otherwise unremarkable. Ten milligrams of aspirin was administered to the baby from 8 days of age to prevent the formation of an intracardiac thrombus.

Catheterization performed at 5 months of age demonstrated normal pulmonary and ventricular pressures including left ventricular end-diastolic pressure and no intracardiac shunt. Angiography of the pulmonary artery revealed a large LAAA in the superior-leftward position to the LA; contractility was preserved in the LAAA (Figure 4). Coronary angiography revealed slight displacement of the left circumflex coronary artery due to mass effect of the aneurysm; nevertheless, no coronary stenosis was noted.

At 7 months of age, the patient underwent surgical resection of the LAAA. The procedure was performed through a median sternotomy with cardiopulmonary bypass to reduce the risk of intracardiac thrombus formation. Intraoperatively, the LAAA was found to extend along the lateral aspect of the left ventricle. The wall of the LAAA was thin and translucent. The LAAA was completely resected, and throughout the procedure, care was taken to avoid obstructing the orifices of the left upper pulmonary vein and the left coronary artery. No thrombus and inflammatory signs were observed in the LAAA. Pathological evaluation of the resected specimen demonstrated thinning of the atrial wall in the subendocardium and pericardium, and patchy interstitial fibrosis of the endocardium and the epicardium (Figure 5). The postoperative course 
was uneventful, and the patient was discharged without any complications. The computed tomographic images revealed that there was no recurrence of the LAAA. Aspirin was discontinued one year later. At 5 years of age, the patient is currently asymptomatic, has achieved normal developmental milestones, and has experienced no recurrence of the aneurysm.

Discussion

Once an LAAA is diagnosed, surgical resection is recommended even for asymptomatic cases, because an increase in the size of the aneurysm over time leads to an elevated risk of arrhythmia and thrombotic events (2). Surgical resection is safe and has been reported to have good outcomes. An LAAA is believed to result from congenital dysplasia of the musculi pectinati (6). Prenatal diagnosis of LAAAs is rarely made, and it is notable that cor triatriatum was suspected on the first fetal echocardiography in the present case. LAAAs should form part of the differential diagnoses in cases where the abnormal chamber is located adjacent to the LA (2). There are no evidence-based recommendations regarding the timing of cardiac surgery, especially for asymptomatic pediatric patients. In the present case, the patient underwent an elective surgery at 7 months of age. It is reported that open heart surgery with cardiopulmonary bypass in the neonatal period involves the risk of neurodevelopmental delay (7). Besides, LAAA resection performed in the neonatal period requires postoperative extracorporeal cardiopulmonary bypass, according to previous reports (8). Furthermore, an LAAA is rarely symptomatic in the first decade of life. Although it grows in size over several years, it can sometimes manifest clinically in young patients including fetuses $(4,5,9,10)$. Based on these facts, elective surgery was selected at 7 months of age to reduce the risk of neurological damage as much as possible. It is suggested that the resection of an LAAA can be performed electively later than in the infancy period in asymptomatic neonatal patients although further research is needed to clarify the optimal timing of surgery in asymptomatic pediatric patients with LAAAs.

Acknowledgments

Informed consent was obtained in the form of opt-out on the website of Osaka City General Hospital.

This study was approved by the Institutional Review Board of Osaka City General Hospital.

I wish to thank Dr. Miho Sakaida for advice on pathological evaluation.

Conflict of Interest

None.

Disclosures

None.

Author contributions

TS designed the study and drafted the manuscript. YK, YM, MF, KN, YY, TS, and KN critically revised the manuscript for intellectual content. EE finally approved the manuscript as a senior author. All authors have read and approved the manuscript.

\section{References}

Yanli Z, Xiaocong, Liping P, et al: Diagnosis of a giant left atrial appendage aneurysm by contrast-enhanced echocardiography: Case report and literature review. J Clin Ultrasound 2021:49:293-297.

Aryal MR, Hakim FA, Ghimire S, et al: Left atrial appendage aneurysm: a systematic review of 82 cases. Echocardiography 2014:31:1312-1318.

Chen Y, Mou Y, Jiang LJ, et al: Congenital giant left atrial appendage aneurysm: a case report. J Cardiothorac Surg 2017:12:15.

$\mathrm{Su}$ X, Yang F, Yu D, et al: Congenital left atrial appendage aneurysm: Prenatal diagnosis and outcome of a rare cardiac abnormality. Echocardiography 2021: 38:480-483. 
Cho MJ, Park JA, Lee HD, et al: Congenital left atrial appendage aneurysm diagnosed by fetal echocardiography. J Clin Ultrasound 2010:38:94-96.

Chowdhury UK, Seth S, Govindappa R, et al: Congenital left atrial appendage aneurysm: a case report and brief review of literature. Heart Lung Circ 2009:18:412-416.

Stolwijk LJ, Lemmers PM, Harmsen M, et al: Neurodevelopmental outcomes after neonatal surgery for major noncardiac anomalies. Pediatrics 2016:137:e20151728.

Clarke JR, Zvaigzne CG, Disler D, et al: Giant Left Atrial Appendage Aneurysm in a Neonate. World J Pediatr Congenit Heart Surg 2012:3:392-395.

Tandon R, Arisha MJ, Nanda NC, et al: Incremental benefit of three-dimensional transthoracic echocardiography in the assessment of left atrial appendage aneurysm leading to severe extrinsic compression of a coronary artery. Echocardiography 2018:35:685-691.

Sarin SS, Bindra T, Chhabra GS. A giant left atrial appendage aneurysm with a large pinball-like thrombus in a 2 year old. Ann Pediatr Cardiol 2012:5:215-216.

Figure legends

\section{Figure 1}

i) The LAAA (abnormal chamber) is adjacent to the LA (arrow). ii) The LAAA is adjacent to the main PA (arrow).

iii) The LAAA had a "to and fro" communication with the LA.

Ao, aorta; LA, left atrium; LAAA, left atrial appendage aneurysm; LV, left ventricle; PA, pulmonary artery; RA, right atrium; RV, right ventricle; SVC, superior vena cava.

\section{Figure 2}

i) Transthoracic echocardiography showing a giant left atrial appendage aneurysm (blue arrow) compressing the left ventricle and LUPV. ii) No flow acceleration was seen at the entry of the LUPV (yellow arrow) to the LA.

LA, left atrium; LUPV, left upper pulmonary vein; LV, left ventricle.

\section{Figure 3}

A three-dimensional view showing a huge left atrial appendage aneurysm on computed tomography angiography.

LAAA, left atrial appendage aneurysm; LUPV, left upper pulmonary vein; LV, left ventricle; RV, right ventricle.

\section{Figure 4}

Angiography of the pulmonary artery. The communication between the left atrium and the left atrial appendage aneurysm (arrow) is evident.

LV, left ventricle.

\section{Figure 5}

Mild fibrotic aneurysmal wall existing with superimposed secondary chronic inflammatory reaction. a) Hematoxylin and eosin stain x100. b) Masson trichrome stain x100. Solid line epicardium, dotted line myocardial layer, doubled line endocardium. 

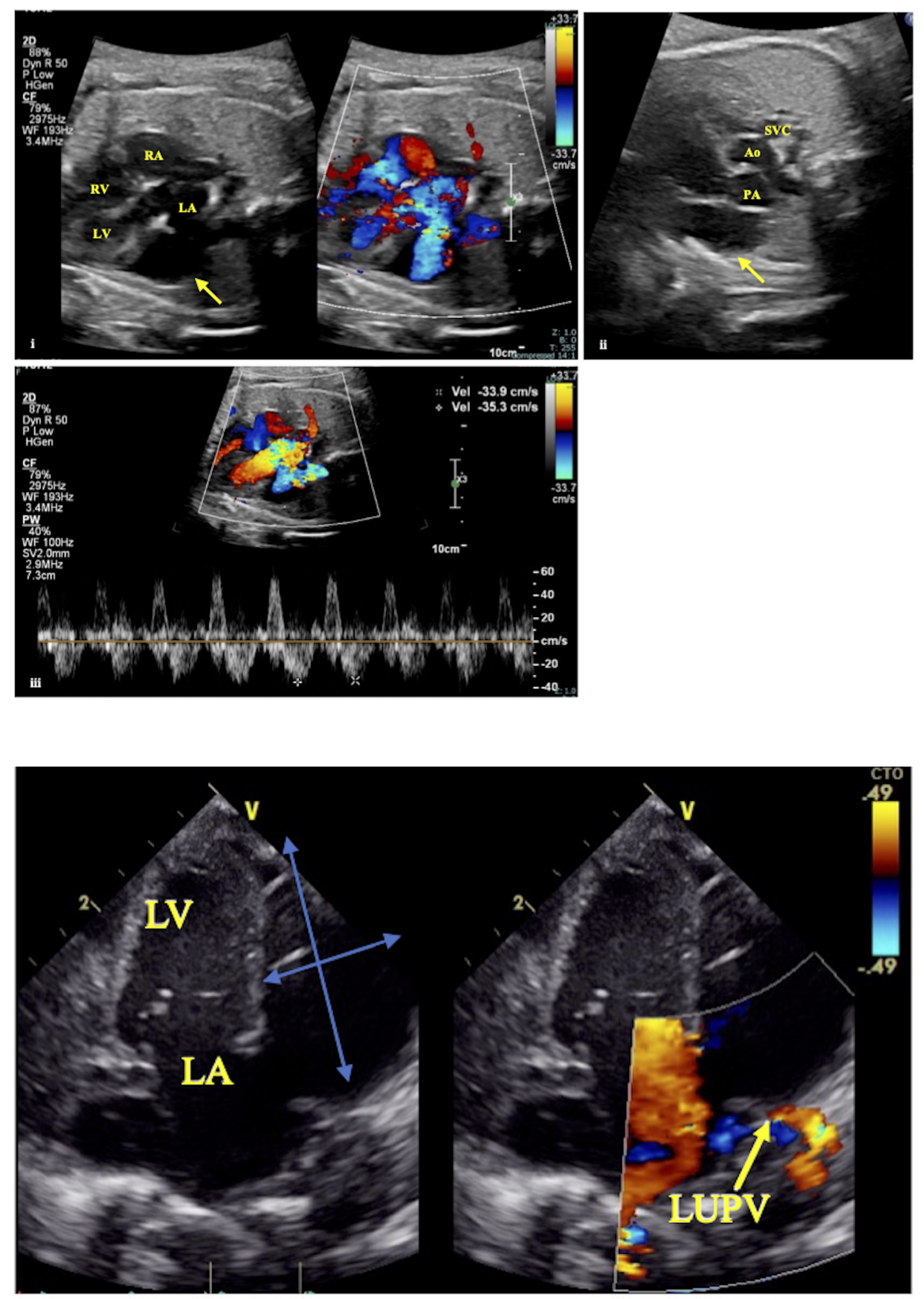

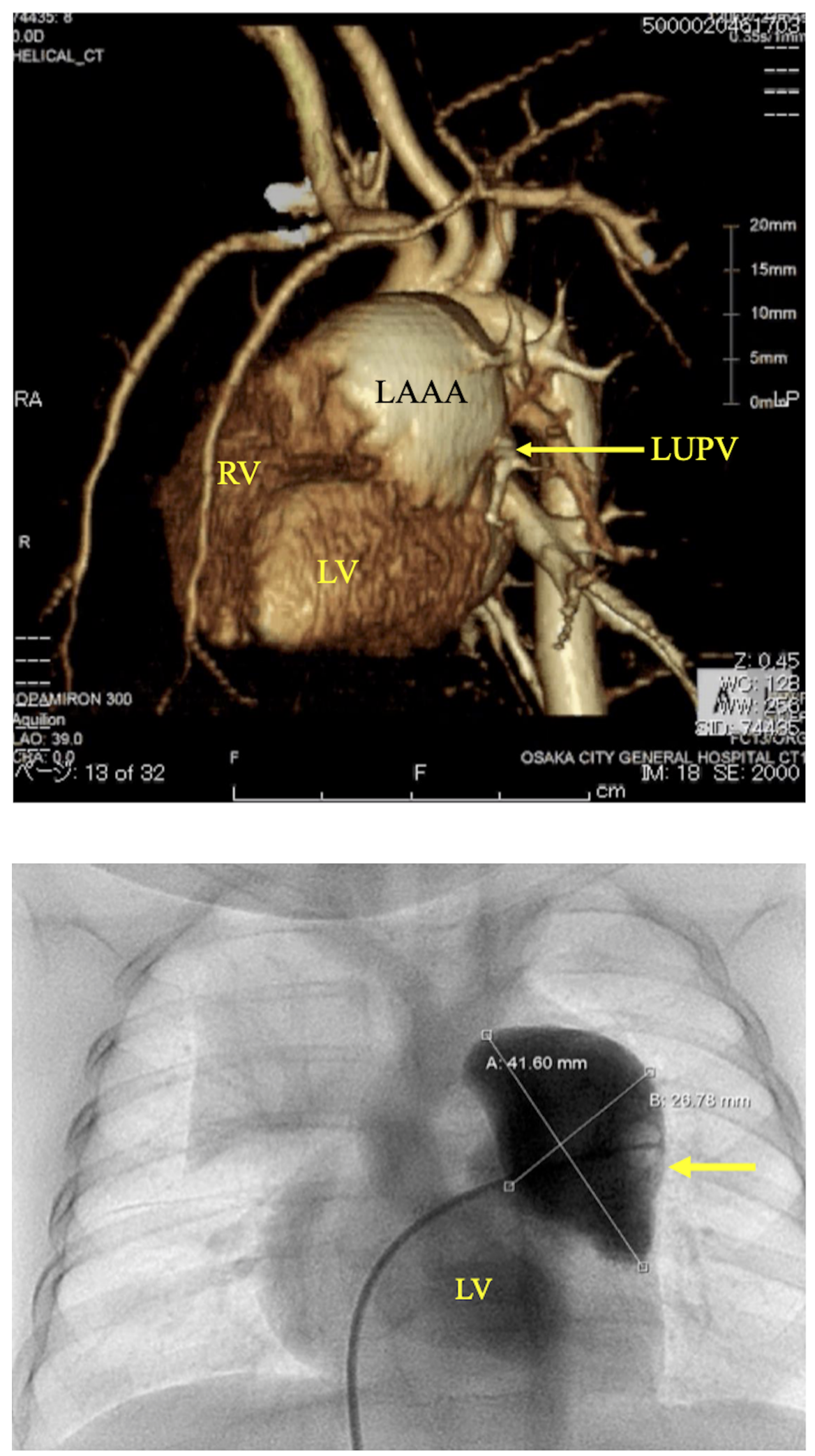


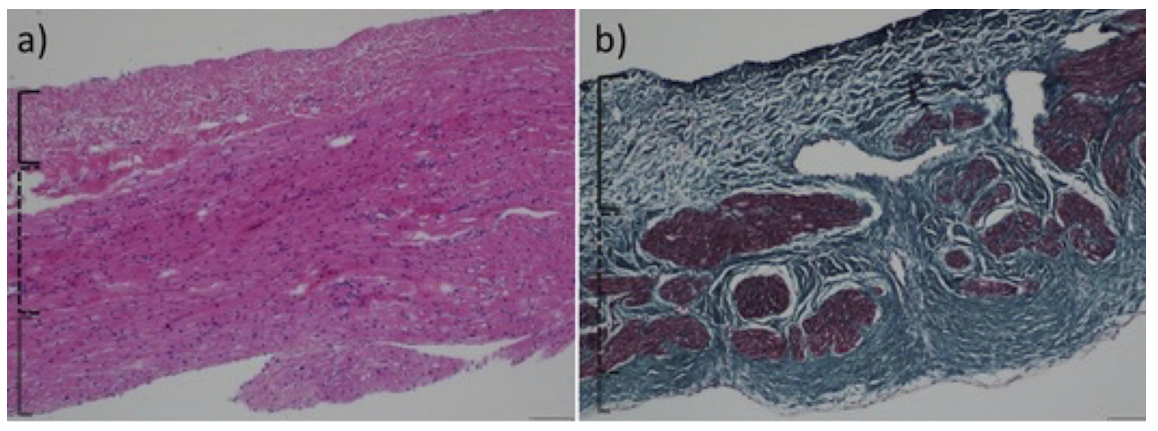

7 